\title{
DEVELOPMENT, MODELLING AND EVALUATION OF A (LAMINAR) ENTRAINED FLOW REACTOR FOR THE DETERMINATION OF THE PYROLYSIS KINETICS OF POLYMERS
}

\author{
R.W.J. WESTERHOUT*, J.A.M. KUIPERS, W.P.M. VAN SWAAIJ \\ Twente University, Reaction Engineering Group, P.O.Box 217.7500 AE ENSCHEDE, The Netherlands
}

\begin{abstract}
Laminar Entrained Flow Reactors were examined to determine whether this type of reactor can be used to measure the kinetic parameters of the pyrolysis reaction of polymers. In case the EFR was operated in the turbulent regime or the diameter of the reactor was to small, sticking of polymer to the reactor wall, became a major problem. In the laminar flow regime this problem did not occur and this operation regime was determined as a function of the Reynolds number. Due to the necessity of operation in the laminar regime significant temperature and velocity gradients exist in the EFR. To correct for these gradients a model was developed incorporating the Navier - Stokes equations to describe the gas phase velocity and temperature distributions and a single particle model to describe the conversion of the individual particles. While correction of the experimental data for the axial gradients proved to be possible. it was not possible to correct this data for radial gradients in the reactor due to the uncertainty in the radial position of the particle. Experiments were performed and corrected for the aforementioned gradients to obtain the first order kinetic parameters for the pyrolysis of LDPE. However, these parameters are inaccurate and therefore a LEFR is preferably not to be used to determine kinetics of particles, if operation of the EFR in the laminar regime is necessary (sticking particles). If possible (non sticking particles) the EFR should be operated in the turbulent regime. Finally our pyrolysis experiments of LDPE showed that intermediate wax - like products are produced during the pyrolysis reaction, which are pyrolysed further in the gas phase.
\end{abstract}

\section{Introduction.}

In the Netherlands alone some $780 \mathrm{kton} / y e a r$ of Mixed Plastic Waste (MPW), mainly consisting of PolyEthene (PE), PolyPropene (PP), PolyStyrene (PS) and PolyVinylChloride (PVC) is produced every year (Rijpkema et al., 1992). Because dumping and incineration of MPW are, or will be forbidden in Germany and the Netherlands, universities and companies are developing alternatives for the disposal of MPW. One of the most promising alternatives is high temperature pyrolysis, by which valuable chemicals as ethene, propene, styrene and $\mathrm{HCl}$ can be produced. Because of the lack of kinetic data in the literature for higher temperatures $\left(>450^{\circ} \mathrm{C}\right.$ ) (Westerhout et al.,1996) it is very difficult to design, scale - up or evaluate reactors for these high temperature pyrolysis processes. The aim of this study was to examine the use of Laminar Entrained Flow Reactors (LEFR) to determine the pyrolysis kinetics of the aforementioned polymers at higher temperatures. This type of reactor has often been used to study the kinetics of the pyrolysis of coal and wood (Ranke, 1990, Wagenaar, 1994, Solomon et al., 1986), but to the knowledge of the authors has not yet been applied to study the pyrolysis kinetics of polymers. However, the use of such a reactor to study the high temperature pyrolysis kinetics of polymers, seems logical, because LEFR's are relatively easy to develop and use.

\section{Equipment and experimental procedures.}

Two Entrained Flow Reactors (EFR) were used in this study. The first EFR was previously used by Wagenaar (1994) to study the kinetics and products of pine wood pyrolysis. His reactor has a diameter of $20 \mathrm{~mm}$ and a length of 0.4 to $1.2 \mathrm{~m}$. In contrast with the reactor shown in figure 1 there was no flow distributor in the entrance section. The second reactor, which was an improved version of the first reactor, has a diameter of $50.4 \mathrm{~mm}$ and a length of $1 \mathrm{~m}$ and incorporates a flow distributor in the entrance section as shown in figure 1. Small polymer particles (diameter $100 \mu \mathrm{m}$ or less) were fed to the reactor through a water cooled inlet pipe, usually called the cold finger. A small cold nitrogen stream was fed with the particles through the cold finger to prevent hot gas from entering the feeding apparatus and the cold finger, which would cause melting and clogging of the polymer in the cold finger. The cold finger was designed so that it could be positioned inside or outside the reactor. The inside diameter of the cold finger, which was chosen as small as possible, was $3.5 \mathrm{~mm}$, while the outside diameter was $12 \mathrm{~mm}$. The cold finger was cooled through the cooling mantle using cold water.

The main nitrogen stream was preheated to the reactor temperature using an electrical tube furnace. The temperature of this stream was monitored with two $\mathrm{K}$ - type thermocouples positioned near the entrance of the reactor. At the outlet of the reactor the pyrolysis products were quenched by injection of a cold nitrogen stream. Unconverted polymer was collected in the bottom section of the jar below the reactor, while intermediate wax - like components were collected in the filters in the top section of the jar. Before and after each experiment the amount of polymer in the feeding apparatus was determined by weighing. The bottom section of the jar and the filters were also weighed before and after each experiment to determine the fractions of unconverted polymer and wax - like products. On the

\footnotetext{
* To whom correspondence should be adressed.
} 
basis of these measurements the conversion of polymer to different products could be calculated with a mass balance.

To determine the extent of polymer sticking to the reactor wall, experiments were conducted at $300^{\circ} \mathrm{C}$. At this temperature the polymer melts, but does not degrade. In this situation all polymer material fed to the reactor should be recovered at the bottom of the jar below the reactor. Experiments with the first reactor as used by Wagenaar (1994) showed that almost no polymer was recovered if the reactor was operated in the turbulent or laminar regime, which implies that significant sticking of polymer material to the reactor wall occurred.

This was confirmed by visual observation of the reactor wall after the experiments. It was also observed that a large amount of the polymer stuck to the reactor wall in the first $100 \mathrm{~mm}$ of the reactor on the side opposite to the gas inlet due to poor design of the inlet section. The rest of the polymer was deposited equally over the reactor wall.

On the basis of the experiments at 300 " $\mathrm{C}$ the following conclusions were drawn :

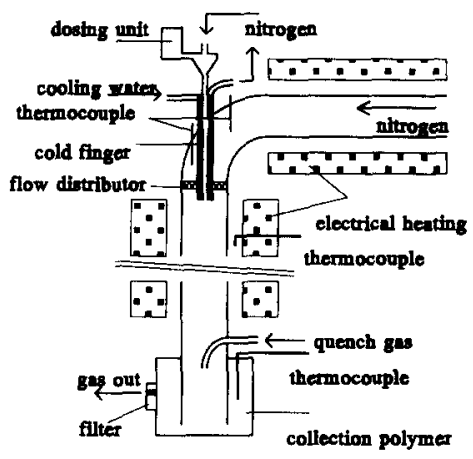

Figure 1: Schematical drawing of Entrained Flow Reactor used in study.

- The reactor should be operated in the laminar regime to reduce radial dispersion and subsequent sticking of polymer to the wall.

- The entrance section of the reactor as used by Wagenaar (1994) should be redesigned to ensure that there are no large radial velocity components at the entrance by which particles are swept to the reactor wall.

- The diameter of the reactor must be enlarged to reduce the chance of sticking of particles to the wall.

After these preliminary experiments with the first reactor, it was decided to develop an improved EFR for this study. Before constructing this reactor cold flow experiments were performed in a glass model to determine the flow regime (in terms of gas velocity), within which the amount of sticking was acceptable.

\section{Hydrodynamic experiments.}

To study the flow behaviour of the particles and gas in the reactor, a glass model of the new reactor was constructed. In this glass model (diameter $50.4 \mathrm{~mm}$, length $1007 \mathrm{~mm}$ ) the prevailing flow regime (turbulent or laminar) was determined for several combinations of gas flows injected through the cold finger and flow distributor. Visualisation of the flow pattern was achieved by injecting smoke into the nitrogen stream fed through the cold finger. Significant dispersion of the smoke was associated with turbulent flow whereas the absence of smoke dispersion was interpreted as the existence of laminar flow. The results of these experiments are presented in figure 2, where the flow regime can be determined as a function of the Reynolds number Re and the ratio $\psi$ between the average velocity of the gas stream passing through the cold finger and the average gas velocity fed through the flow distributor.

As evident from figure 2 the flow regime in the reactor strongly depends on the velocity ratio $\psi$ and the Reynolds number Re. At low Reynolds numbers the flow remains laminar, even if the disturbance of the flow due to the injection of the nitrogen through the cold finger is large (large $\psi$ ), but at higher Reynolds numbers the nitrogen injected through the cold finger changes the flow regime in the reactor from laminar to turbulent even at low velocity ratios. These results are consistent with the results found by Flaxman et al. (1987), although these authors experimented at higher Reynolds numbers and generally with lower velocity ratios.

To study the effect of the Reynolds number and the velocity ratio on the radial distribution of particles in the EFR, cold flow experiments were carried out with LDPE particles. The particles were collected at the end of the EFR. Particles falling through the middle of the reactor $(\mathrm{r} / \mathrm{R}<0.7)$ were separately collected from particles, which could potentially stick to the reactor wall under actual reactor conditions ( $r / R>0.7)$. In the experiments the Reynolds number was varied by altering the velocity through the flow distributor. During these experiments the velocity ratio 
$\Psi$ was therefore not kept constant, but only the velocity through the cold finger. Some results of these experiments are presented in figure 3 .

From this figure it can be concluded, that a EFR should preferably be operated at low Reynolds numbers and additionally that the nitrogen flow through the cold finger should be kept as low as possible. In the optimal situation $\left(\mathrm{u}_{\mathrm{cF}}<0.11 \mathrm{~m} / \mathrm{s}\right.$ and $\left.\mathrm{Re}<100\right)$ the fraction of polymer which will come close enough to the reactor wall to possibly stick to it can be reduced to less than $5 \%$. Of course a much smaller fraction will actually stick to the reactor wall, so that the loss of polymer material due to sticking to the tube wall is acceptable in this situation.

Our experiments also showed that the radial distribution of polymer particles was independent of the reactor length. However, the length of the reactor should not be chosen to long, because the extent of polymer sticking to the reactor wall will increase in longer EFR's, since more time and area is available to the particles to stick to the tube wall.

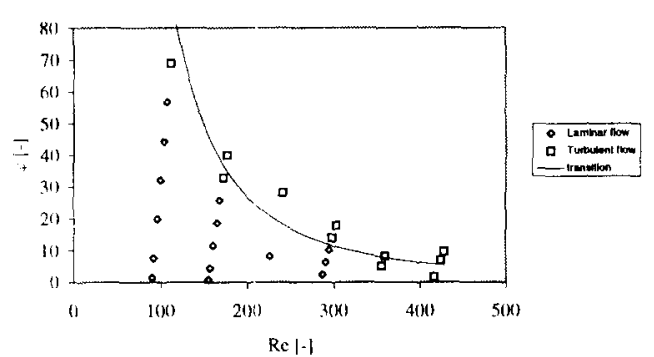

Figure 2 : Operation regime of EFR as a function of Figure 3 : Fraction of polymer falling near the tube wall velocity ratio $\psi$ and Reynolds number $R e$.

Experiments at 300 " $\mathrm{C}$ with a LDPE confirmed the results of the cold flow experiments. The extent of sticking in the new LEFR operated at actual reactor conditions (low Reynolds numbers and a low velocity through the cold finger) was negligible. The experiments described in this paragraph have shown that the use of a LEFR is potentially possible from a hydrodynamic point of view, provided that the LEFR is properly designed and operated.

\section{Model.}

To prevent sticking of polymer to the reactor wall an EFR has to be operated in the laminar regime. In this flow regime radial and axial temperature and velocity gradients will exist inside the reactor due to entrance effects and the injection of the cold nitrogen stream through the cold finger. To predict these gradients a model has been developed to enable prediction of the influence of these gradients and to correct the kinetic measurements for these effects.

An excellent review of the models used in the literature was recently given by Flaxman et al. (1987). The models usually consist of two parts : one part to describe the gas phase and a second part to describe the pyrolysis of individual particles.

The most elaborate models were published by Tsai et al. (1984) and Jamaluddin et al. (1986). These authors used the Navier - Stokes equations to calculate the temperature and velocity distributions in the gas phase.

In the model of Tsai et al. (1984), the density of the gas phase was assumed to be constant, an assumption which holds for the main preheated gas stream, but which certainly is not valid for the cold gas stream injected through the cold finger. The temperature change of the gas stream will cause a volumetric expansion of a factor 2 or more, which probably significantly influences the axial temperature and velocity in the centre of the reactor. No information was given in their article, about the boundary conditions used (influence of the presence of the cold finger). There is no indication in their article, that the model is able to predict the conversion of individual particles as a function of the axial position. Another elaborate model was published by Weeda (1995), who used the FLUENT CFD package to compute the temperature and velocity distributions in a LEFR. Apparently this model also does not include a particle 
mass balance to calculate the conversion of individual particles, which limits its applicability. In the article of Jamaluddin et al. (1986) almost no information is given regarding the simplifications used in the gas phase model or the applied boundary conditions. However, their model was used to predict the conversion of individual particles as a function of the axial position.

The model used in this work is an expansion of the model of Tsai et al. (1984), because two of their simplifications are not used in this study : the gas phase density is not assumed constant and the influence of the cold finger is accounted for in the boundary conditions, while reaction in the particle is included. It is also possible with our model to calculate the temperature gradients inside the particle to check, whether internal heat transfer resistances are of importance.

The following main assumptions are used in our model :

- The gas phase flow is laminar.

- The gas phase velocity distribution is not altered due to the presence of the particles (one - way coupling).

- The gas phase temperature distribution is not influenced by the endothermic pyrolysis process of the polymer particles.

- The gas phase is transparent with respect to thermal radiation. At very high temperatures $\left(>1000^{\circ} \mathrm{C}\right)$ this assumption may not hold.

Assuming that the flow in the reactor is laminar and is not influenced by the particles, the gas phase axial and radial temperature and velocity distributions can be calculated using the Navier - Stokes equations in axi - symmetrical cylindrical co-ordinates (Bird et al., 1960). The assumption that the gas flow is laminar can be checked with figure 2. The particle loading is kept sufficiently low to ensure that the gas phase is not exhausted by the endothermic pyrolysis reaction, so that the interaction between the particles and the gas phase can be neglected.

The gas phase flow is not developed when entering the reactor (flat velocity profile), while two streams with different velocities and temperatures are mixed (temperature profiles), so that gradients will exist in the reactor. The Navier Stokes equations are used to calculate these gradients in the reactor :

Continuity equation :

$$
\frac{\partial p_{f}}{\partial t}+\frac{1}{r} \frac{\partial}{\partial r}\left(r \rho \mu_{r}\right)+\frac{\partial}{\partial z}\left(p_{\mu} \mu_{z}\right)=0
$$

Equation of motion, $r$ - component :

$$
\frac{\partial}{\partial t}\left(p \mu_{r}\right)+\frac{1}{r} \frac{\partial}{\partial r}\left(r p \mu_{r} u_{r}\right)+\frac{\partial}{\partial z}\left(p \mu_{z} u_{r}\right)=-\frac{\partial P}{\partial r}-\left[\frac{1}{r} \frac{\partial}{\partial r}\left(r \tau_{r}\right)-\frac{\tau_{00}}{r}+\frac{\partial \tau_{n}}{\partial z}\right]+p g,
$$

Equation of motion, z - component :

$$
\frac{\partial}{\partial t}\left(p \mu_{x}\right)+\frac{1}{r} \frac{\partial}{\partial r}\left(r p_{\mu} u_{z}\right)+\frac{\partial}{\partial z}\left(\rho \mu_{s} u_{s}\right)=-\frac{\partial P}{\partial z}-\left[\frac{1}{r} \frac{\partial}{\partial r}\left(r \tau_{r z}\right)+\frac{\partial \tau_{z z}}{\partial z}\right]+\rho \mathcal{B}_{z}
$$

The components of the stress tensor $\tau\left(\tau_{\pi}, \tau_{\theta \theta}, \tau_{z z}\right.$ and $\left.\tau_{r z}\right)$ are assumed to obey the general Newtonian fluid form (Bird et al., 1960). In the thermal energy equation for the gas phase the viscous dissipation term has been neglected, which is allowed for systems without large velocity gradients (Bird et al., 1960). 


$$
\frac{\partial}{\partial t}(p / f)+\frac{1}{r} \frac{\partial}{\partial r}\left(r p_{f} f_{r}\right)+\frac{\partial}{\partial z}\left(p / \mu_{z}\right)=-\left[\frac{1}{r} \frac{\partial}{\partial r}\left(r q_{r}\right)+\frac{\partial q_{z}}{\partial z}\right]-p\left[\frac{1}{r} \frac{\partial}{\partial r}\left(r u_{r}\right)+\frac{\partial u_{z}}{\partial z}\right]
$$

The boundary conditions required to solve the gas phase balance equations are summarized in figure 4 . In our model the actual geometry of the LEFR is used. This constitutes an important feature of our model, since the geometry has an important effect on the prevailing velocity and temperature distributions. The calculated steady state gas phase velocity and temperature distributions are used as dynamic boundary conditions for the single particle model, which is used to calculate the position, velocity and conversion of the particle and the intraparticle temperature.

Our single particle model assumes a constant particle diameter and incorporates balance equations 5,6 and 7.From equations 7 and 8 both the radial and axial position can in principle be calculated. The calculation of the radial position requires a number of additional assumptions. However 'as a first approximation' we assumed in this study that the particles travel along the centre line of the LEFR, which implies that only the axial components of equations 7 and 8 were solved.

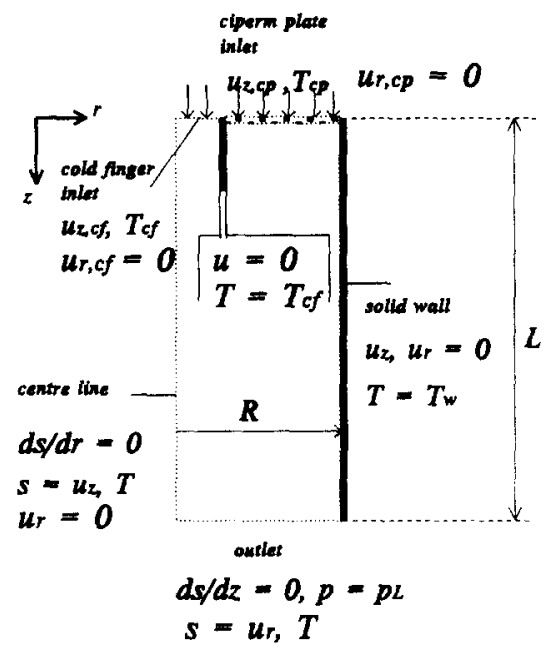

Figure 4 : Schematic representation of computational domain and boundary conditions.

Mass balance :

$$
\frac{\partial \xi}{\partial t}=k_{0} e^{-\frac{E_{e x t}}{R_{s} r}}(1-\xi)
$$

Enthalpy balance :

$$
p_{p, 0}(1-\xi) c_{p p} \frac{\partial T}{\partial t}=\frac{1}{x^{2}} \frac{\partial}{\partial x}\left[\lambda_{p} x^{2} \frac{\partial T_{p}}{\partial x}\right]-k_{0} e^{-\frac{\xi_{e}}{\lambda_{p} T_{r}}}(1-\xi)^{n} p_{p, 0} \Delta H_{r}
$$

Force balance :

$$
P_{p, 0}(1-\xi) V_{p} \frac{d \overline{v_{p}}}{d t}=V_{p}\left(p_{p, 0}(1-\xi)-p_{p} \bar{g}+\frac{1}{2} C_{d} p_{f} \bar{u}-\overline{v_{p}}\left(\bar{u}-\overline{v_{p}}\right) A_{p}\right.
$$

Differential equation to calculate particle position vector :

$$
\overline{v_{p}}=\frac{d \bar{r}}{d t}
$$

In addition to the boundary conditions shown in figure 4 , there is also a boundary condition required at the particle gas phase interface for which the following mixed boundary condition has been used : 


$$
\left.\lambda_{p} \frac{\partial T_{p}}{\partial x}\right|_{x_{p}}=\alpha\left(T_{f}-T_{x_{p}}\right)+\epsilon * \sigma *\left(T_{w}^{4}-T_{x_{p}}^{4}\right)
$$

To solve the model constitutive relations are needed for $\alpha$ and $C_{\mathrm{d}}$. To calculate the external heat transfer coefficient $\alpha$ the well - known Ranz - Marshall equation was used, while for drag coefficient well established empirical correlations were incorporated. In the model $c_{p, p}, \mu_{f}$ and $\lambda_{f}$ are considered to depend on the temperature (Vargaftik, 1975, Bandrup et al., 1989), while $\rho_{\mathrm{f}}$ is calculated using the ideal gas law.

Both model parts (gas phase model and single particle model were validated by comparing the numerical solutions of the model parts with available analytical solutions for special cases. With the model described in this paragraph model runs were performed to examine the influence of different parameters on the operation of the reactor and the interpretation of the experimental results. The results of these model runs are presented in the next paragraph.

\section{Results of model runs.}

To evaluate the influence of the different parameters a standard case for the $50 \mathrm{~mm}$ diameter LEFR was defined. The parameters used in this standard case correspond to the typical operation conditions during an experiment and are listed in table 1.

\begin{tabular}{|c|c|}
\hline Parameter & Value \\
\hline $\mathrm{T}_{\mathrm{cI}}$ & $293 \mathrm{~K}$ \\
$\mathrm{~T}_{\mathrm{w}}\left(\mathrm{T}_{\mathrm{cp}}\right)$ & $823 \mathrm{~K}$ \\
$\mathrm{u}_{\mathrm{x}, \mathrm{ci}}$ & $0.73 \mathrm{~m} / \mathrm{s}$ \\
$\mathrm{u}_{\mathrm{z}, \mathrm{cp}}$ & $0.34 \mathrm{~m} / \mathrm{s}$ \\
$\mathrm{X}_{\mathrm{p}}$ & $0.030 \mathrm{~mm}$ \\
$\operatorname{Re}$ & 194 \\
$\Psi$ & 2.2 \\
\hline
\end{tabular}

Table 1: Parameter values for the standard case.

The gas velocity through the cold finger was chosen as low as possible, whereby the clogging of the cold finger was the limiting factor. The required velocity to prevent clogging was determined experimentally. After several tests the cold finger was positioned inside the reactor (30 $\mathrm{mm}$ axial separation of tip from the distributor) to prevent the polymer from hitting and melting in the distributor at the reactor entrance. In our simulations the actual reactor geometry was used.

In figure 5 the axial component of the gas phase velocity is shown as a function of the radial position for several axial locations, whereas in figure 6 the corresponding data for the gas phase temperature is presented.

The gas phase velocity and temperature gradients can not be neglected when interpreting experiments as can be seen from figures 5 and 6 . Even after 1 meter the flow is still developing, although the deviation from the stationary velocity and temperature profiles is the most significant within the first 0.3 meter of the reactor. One major feature of the velocity profile is the local minimum near the entrance. This phenomenon is due to the presence of the cold finger which occupies a region through which no gas can enter the reactor. Additionally, the gas entering through the cold finger has to be heated up from the ambient temperature $(298 \mathrm{~K})$ to the reactor temperature, causing a large volumetric expansion of the flow. These two effects cause the large velocity gradients and the local minimum near the entrance section of the reactor. 

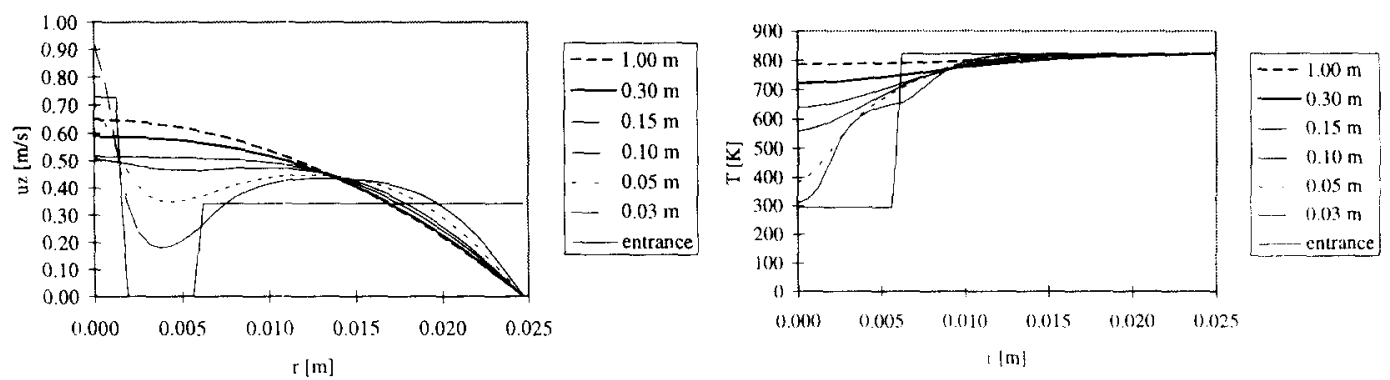

Figure 5: Axial component of the gas phase velocity as a Figure $6:$ Gas phase temperature as a function of the function of the radial position. radial position for several axial locations.

Additional calculations were preformed for a LEFR of $20 \mathrm{~mm}$ diameter corresponding to the LEFR used by Wagenaar (1994) (nol shown here). In the $20 \mathrm{~mm}$ LEFR the disturbance caused by the cold finger was more significant due to its larger relative size, but the distance over which the gradients have to be reduced is smaller in this case. Because of the smaller gradients, the diameter of the LEFR should be chosen as low as possible, but the minimal permissible diameter is determined by the requirement that no polymer particles deposit on the wall during the experiments. To minimize particle deposition on the wall, the LEFR should not only be operated in the laminar regime to prevent radial dispersion of the particles, but it should also posses a minimum diameter of $50 \mathrm{~mm}$. Both requirements enlarge the velocity and temperature gradients in the LEFR.

Correction for the axial gradients is possible as the axial position of the particle can be calculated accurately at all times using the model. but correction for the radial gradients is more difficult due to the uncertainty in the radial position of the particle. Especially the temperature gradients are very important, because pyrolysis reactions have relatively high activation energies and therefore even a small temperature change results in a large change in reaction rate. For this reason the influence of the temperature gradients is more pronounced than the influence of the velocity gradients. Due to the fact that the gas phase temperature at the particle interface can not be accurately predicted, which is especially caused by the uncertainty in the radial position of the particle, significant interpretation errors may be made.

The influence of the temperature and velocity profiles on the conversion profile can be seen from figure 7 in which two cases are compared. In the first case the gas phase temperature and velocity were assumed constant, while in the second case they were calculated using the Navier - Stokes equations. The influence of the gradients on the conversion and conversion rate is significant as can be seen in figure 7 .

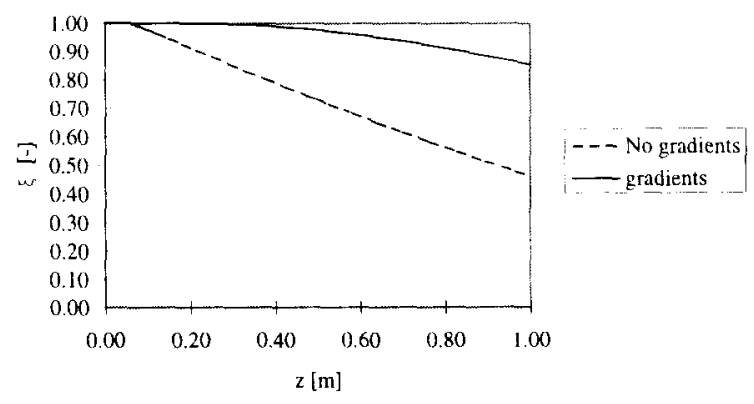

Figure 7 : Influence of temperature and velocity gradients on calculated conversion profile. 


\section{Experimental results.}

The experiments were performed using LDPE 1765 with a density of $917 \mathrm{~kg} / \mathrm{m}^{3}$ and an average molar weight of $350.000 \mathrm{~g} / \mathrm{mol}$. The polymer was sieved to obtain particles with a diameter less than $100 \mu \mathrm{m}$. The gas phase velocity through the cold finger was chosen as low as possible $(0.729 \mathrm{~m} / \mathrm{s}$ at $298 \mathrm{~K}$ and $1 \mathrm{bar})$, while the flow rate of the hot gas injected through the flow distributor was also chosen very low $\left(2.26^{*} 10^{-4} \mathrm{Nm}^{3} / \mathrm{s}\right)$ to ensure laminar flow in the reactor $(\operatorname{Re} 757, \Psi 0.57$, laminar flow, see Flaxman et al. (1987)). During the experiment about 25 to 30 grams of polymer was fed to the LEFR over 90 minutes of operation. Other experimental details are described in paragraph 2. Figure 8 shows the conversion of polymer to gas and wax - like products as a function of the wall temperature, while figures 9 and 10 show the calculated temperature and velocity in the centre of the reactor, which were used to calculate the kinetic constants from these experiments. The velocity in the centre of the reactor increases sharply near the inlet of the reactor from $0.73 \mathrm{~m} / \mathrm{s}$ to $0.93 \mathrm{~m} / \mathrm{s}$, because of the fast development of a parabolic velocity profile in the small cold finger. This effect is not visible in figure 9 because of the scale.

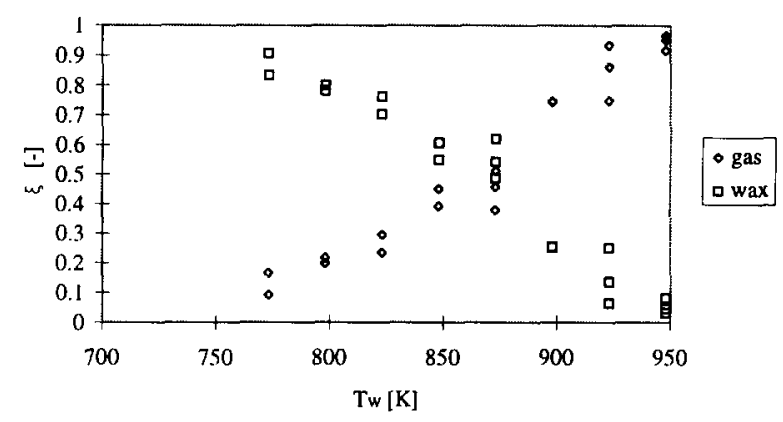

Figure 8: Measured conversion of LDPE to gas and wax - like products as a function of the wall temperature.
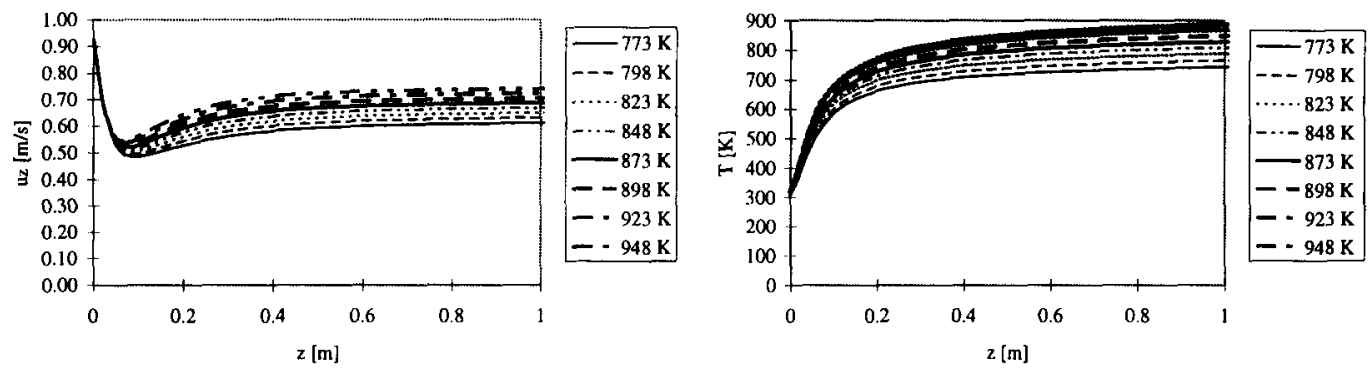

Figure 9: Gas phase velocity in the centre of the reactor Figure 10: Gas phase temperature in the centre of the as a function of axial position. reactor as a function of axial position.

With the model the first order kinetic parameters (i.e. the pre - exponential constant $k_{0}$ and the activation energy $\left.E_{a c t}\right)$ were calculated from the experimental results, which were corrected for external and internal heat transfer limitations. It was assumed that all particles travelled along the centre line of the LEFR. However, in our experiments the internal heat transfer resistance turned out to be negligible because of the usage of small particles. The resulting corrected kinetic parameters are presented in table 2 together with the kinetic parameters determined with a TGA in the temperature range from 400 to 450 " $\mathrm{C}$. 


\begin{tabular}{|c|c|c|}
\hline Method & $\mathrm{k}_{0}\left(\mathrm{~s}^{-1}\right)$ & $\mathrm{E}_{\mathrm{acl}}(\mathrm{kJ} / \mathrm{mol})$ \\
\hline TGA $\left(<450^{\circ} \mathrm{C}\right)$ & $1.0^{*} 10^{15}$ & 241 \\
LEFR $\left(475-575^{\circ} \mathrm{C}\right)$ & $5^{*} 10^{9}$ & 150 \\
\hline
\end{tabular}

Table 2 : Kinetic parameters of LDPE pyrolysis reaction determined with TGA and LEFR.

The kinetic constants measured with a TGA and a LEFR differ significantly as evident from inspection of table 2. One reason for this difference is that the kinetic constants are valid for two different conversion ranges: the TGA kinetic constants are valid for the 70 to $90 \%$ conversion range (Westerhout et al., 1996), while in LEFR experiments usually lower conversions are achieved. However, the main reason for the significant deviation is the large interpretation error made as mentioned in paragraph 5. Due to the radial dispersion the actual average temperature at the particle interface will exceed the temperature used in evaluating the kinetic constants, since in the centre of the LEFR the lowest temperature exists at a fixed axial position.

Closer examination of the solid product collected at the bottom of the LEFR, showed that this product can be separated into unconverted polymer, which is collected at the bottom of the jar and partially converted polymer, wax - like intermediate material which is recovered from the filters in the jar. The relative yields of gas and the wax - like product are shown in figure 8 as a function of the wall temperature. The wax - like product is an intermediate product, which is expected to consist of longer alkanes and alkenes. At the pyrolysis conditions these products exist in the gas phase, but are wax - like solids at ambient conditions. The gas, which is produced is the degradation product of the intermediate wax - like material. The existence of such intermediate materials has been demonstrated by Seeger et al. $(1975,1977)$ and is in accordance with predictions from a recently developed Random Chain Dissociation (RCD) model (Westerhout et al., 1996).

\section{Conclusions.}

It has been possible to design a LEFR in which sticking could be suppressed effectively, which is a necessity when performing kinetic experiments in this apparatus. The calculated kinetic constants are close (in a order of magnitude) to the extrapolated kinetic constants obtained from TGA measurements (Westerhout et al., 1996), which is quite satisfactory when one considers the experimental and interpretation errors, inevitably made when using a LEFR and a simple first order kinetic equation, which is only applicable for a small conversion range. The LEFR can be used to obtain an indication of the reaction rate at a given temperature. The possible error in interpreting the experiments however is large, which is due to radial particle dispersion and the radial temperature gradient for which no correction was applied. Correction for these gradients is in theory possible, but will be difficult in practice due to uncertainties in some model parameters required (initial conditions). If non - sticking particles are studied such as wood and coal particles, the EFR should be operated in the turbulent regime to reduce temperature and velocity gradients. It turned out that for polymer particles this is not possible EFR's, which have to be operated in the laminar regime because of sticking problems, should only be used for determining kinetic constants if no other option is available. The use of an apparatus like a screen heater should be preferred because the control of the temperature and the reactor conditions is much better in this case. If a LEFR is used to study kinetics of a reaction, one should realize that the interpretation error can be significant even if a proper model is used.

\section{Acknowledgments.}

The authors would like to thank Koen Wolf for performing the experiments and computations and Jan Waanders, Wim Leppink and Arno Schanssema for their technical assistance. The authors would also like to express their gratitude to the IOP Recycling and the OSF POWAM projects for financial support of this project.

\section{Notation.}

$\begin{array}{ll}A_{p} & : \text { particle surface area } \\ c_{p} & : \text { heat capacity } \\ C_{d} & : \text { drag coefficient } \\ D & : \text { diameter reactor } \\ E_{i k t} & : \text { activation energy } \\ g & : \text { gravity constant }\end{array}$

$\begin{array}{lr}\left(\mathrm{m}^{2}\right) & \alpha \\ (\mathrm{J} / \mathrm{kg} \cdot \mathrm{K}) & \theta \\ (-) & \epsilon \\ (\mathrm{m}) & \lambda \\ (\mathrm{J} / \mathrm{mol}) & \xi \\ \left(\mathrm{m} / \mathrm{s}^{2}\right) & \mu\end{array}$

$\begin{array}{ll}\text { : external heat transfer coefficient } & \left(\mathrm{W} / \mathrm{m}^{2} \mathrm{~K}\right) \\ : \text { angle coordinate } & (\mathrm{rad}) \\ : \text { emissivity } & (-) \\ : \text { thermal conductivity } & (\mathrm{W} / \mathrm{m} . \mathrm{K}) \\ : \text { conversion, }\left(\rho_{\mathrm{p}, 0}-\rho_{\mathrm{p}}\right) / \rho_{\mathrm{p}, 0} & (-) \\ : \text { viscosity } & (\text { Pa.s })\end{array}$




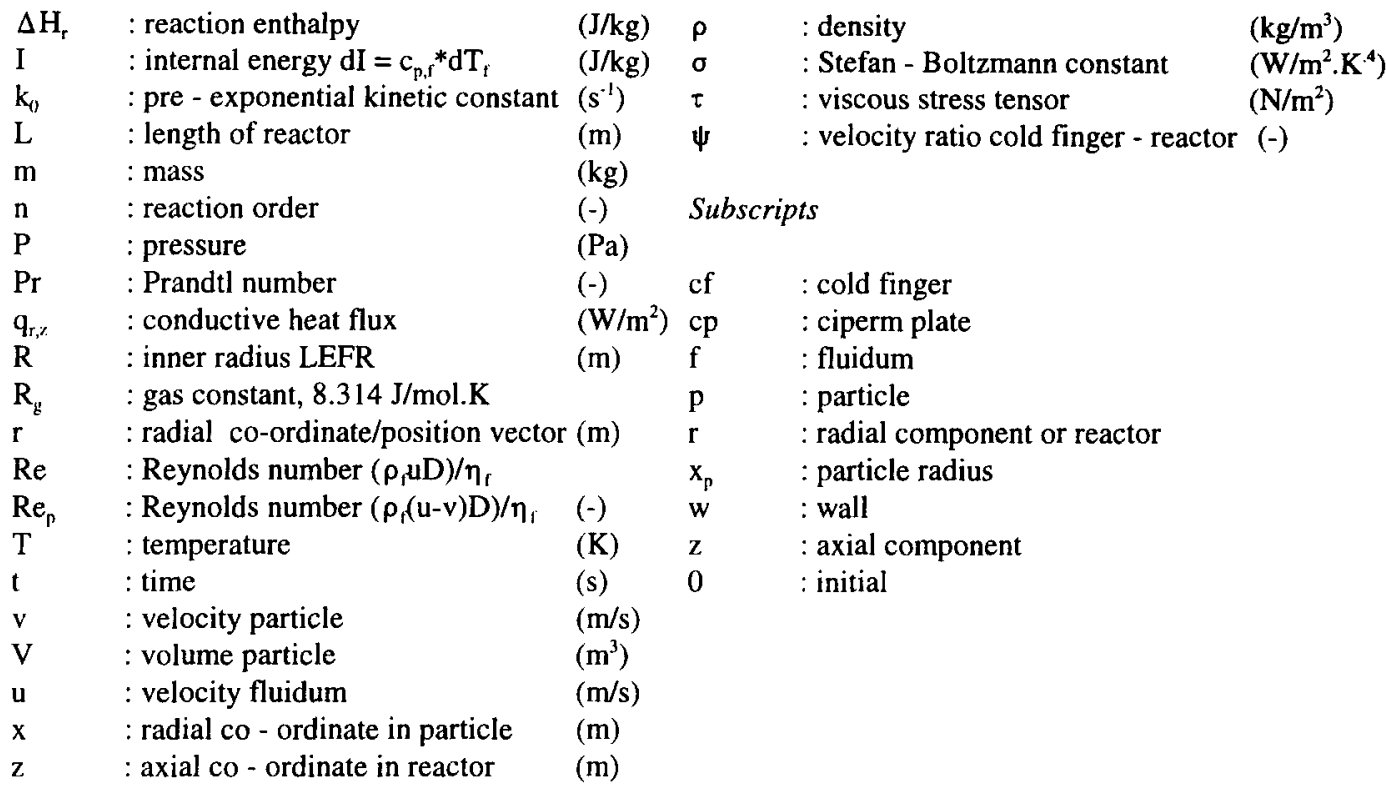

\section{References.}

Bandrup, J. and Immergut, I.H., Polymer Handbook, John Wiley \& Sons, New York, USA, 1989.

Bird, R.B., Stewart, W.E. and Lightfoot, E.N., Transport Phenomena, John Wiley \& Sons, New York, U.S.A., 1960.

Flaxman, R.J. and Hallet, W.L.H., Flow and Particle Heating in an Entrained Flow Reactor, Fuel, Vol. 66, pp. 607 611, 1987.

Jamalludin, A.S., Truelove, J.S and; Wall, T.F., Devolatilization of Bituminous Coals at Medium to High Heating Rates, Combustion and Flame, Vol. 63, pp. 329 - 337, 1986.

Ranke, U., Kinetik der Pyrolyse und der Verbrennung von Steinkohlen unter besonderere Berücksichtigung des Sauerstoffpartialdruckes, Ruhr-Universität, Ph.D. - thesis, Bochum, Essen, Deutschland, 1990.

Rijpkema, L.P.M., Krajenbrink, G.W., Stijnman, P.W.A., Groot, J.L.B. de, Survey of Municipal Solid Waste in Europe, TNO Institute for Environmental and Energy Technology, The Netherlands, 1992.

Seeger, M. and Cantow, H.-J., Thermische Spaltungsmechanismen in Homo-und Copolymeren aus $\alpha$ - Olefinen, Die Macromolekulaire Chemie, Vol. 176, pp. 1411 - 1425, 1975.

Seeger, M. and Ritter, R.J., Thermal Decomposition and Volatilization of Poly( $\alpha$ - olefins), Journal of Polymer Science, Vol. 15, pp. 1393 - 1402, 1977.

Solomon, P.R., Serio, M.A., Carangelo, R.M. and Markham, J.R., Very Rapid Coal Pyrolysis, Fuel, Vol. 65, pp. $182,1986$.

Tsai, C-Y. and Scaroni, A.W., Pyrolysis during the Initial Stages of Pulverized-coal Combustion, $20^{\text {th }}$ symposium (International) on combustion/The Combustion Institute, pp. 1455 - 1462, 1984.

Vargaftik, N., Handbook of Physical Properties of Liquids and Gases (Pure Substances and Mixtures), Hemisphere Publishing Company, Washington, USA, 1975.

Wagenaar, B.M., The Rotating Cone Reactor for Thermal Solids Processing, TU Twente, Ph.D. - thesis, Enschede, The Netherlands, april 1994.

Weeda, M., Kinetics of Coal Gasification under Industrial Conditions. Exploratory Study of the Feasibility of Chemical Cooling in Novel Iron Production Processes, University of Amsterdam, Amsterdam, The Netherlands, 1995.

Westerhout, R.W.J., Kuipers, J.A.M. and Swaaij, W.P.M. van, Experimental Determination and Modelling of the Low Temperature Pyrolysis Kinetics of PolyEthene, PolyPropene and PolyStyrene, to be published, 1996. 